\title{
Engagement e conteúdo em vídeos sobre a aplicação de online dating Tinder no YouTube*
}

Rita Sepúlveda ${ }^{1}$

Recibido: 26/01/2020

Aprobado por pares: 13/04/2020
Enviado a pares: $30 / 01 / 2020$

Aceptado: 31/07/2020

DOI: 10.5294/pacla.2021.24.4.6

Para citar este artículo / to reference this article / para citar este artigo Sepúlveda, R. (2021). Engagement e conteúdo em vídeos sobre a aplicação de online dating Tinder no YouTube. Palabra Clave, 24(4), e2446. https://doi.org/10.5294/pacla.2021.24.4.6

\section{Resumo}

As soluções de online dating têm-se diversificado e crescido em número de utilizadores, potenciadas principalmente pelas aplicações móveis. Entre estas, a aplicação Tinder tem sido objeto de atenção pela imprensa generalista e de estudo pela academia, devido a um conjunto de características distintivas que têm levantado várias questões. Esta investigação propõe uma abordagem metodológica no sentido de compreender que narrativas relativas à aplicação e aos seus utilizadores são disseminadas no YouTube. O YouTube tem-se revelado uma plataforma importante na disseminação de narrativas sobre diversas temáticas, permitindo a partilha de conteúdo próprio. Para tal, e com recurso a métodos digitais, foi realizada uma análise quantitativa e qualitativa aos vídeos no YouTube mais influentes sobre o

Este trabalho foi financiado através de fundos do Ministério da Ciência, Tecnologia e Ensino Superior/Fundação para a Ciência e a Tecnologia, no âmbito da bolsa de doutoramento com a referência SRFRH/BD/130648/2017.

$1 \bowtie$ https://orcid.org/0000-0003-4348-5637. Instituto Universitário de Lisboa (ISCTE-IUL), Centro de Investigação e Estudos de Sociologia, Lisboa, Portugal.racfc@iscte-iul.pt 
Tinder. Os resultados mostraram que estes retratavam fases do processo de online dating como a de criação do perfil, a de correspondência e a de descoberta, refletindo sobre controvérsias associadas à aplicação e sustentandoas. A criação dos vídeos era, na maior parte das vezes, impulsionada pela curiosidade sobre o Tinder e como objeto para criar conteúdo atrativo para a audiência.

\section{Palavras-chave (Fonte: tesauro da Unesco)}

Telefone inteligente; comunicação; online dating; Tinder; YouTube; vídeos. 


\section{Engagement y contenido en videos sobre el aplicativo de citas Tinder en YouTube*}

\section{Resumen}

Las soluciones de online dating se han diversificado y aumentado en número de usuarios, potenciadas principalmente por aplicativos móviles. Entre estas, el aplicativo Tinder ha sido objeto de atención por la prensa generalista y de estudio por la academia, debido a un conjunto de atributos distintivos que han levantado varias cuestiones. Esta investigación plantea un enfoque metodológico en el sentido de comprender qué narraciones relativas al aplicativo y a sus usuarios se diseminan en el YouTube, el que se ha develado una plataforma importante en la diseminación de narraciones acerca de diversas temáticas, lo que permite el compartir de contenido propio. Para tal, y con recurso a métodos digitales, se realizó un análisis cuantitativo y cualitativo de los videos en el YouTube más influenciadores sobre el Tinder. Los resultados evidencian que estos retrataban fases del proceso de online dating, como la de creación del perfil, la de correspondencia y la de descubierta, con reflexiones sobre las controversias asociadas con el aplicativo y sosteniéndolas. La creación de los videos era, mayormente, impulsada por la curiosidad sobre el Tinder y como objeto para crear contenido atractivo para el público.

\section{Palabras clave (Fuente: tesauro de la Unesco)}

Teléfono inteligente; comunicación; online dating; Tinder; YouTube; videos. 


\section{YouTube's Engagement and Video Content about the Online Dating Application Tinder}

\section{Abstract}

Online dating solutions have diversified and increased in the number of users, mainly powered by mobile applications. Among them, Tinder has drawn the attention of the generalist press and academia due to a set of distinctive attributes that have posited several questions. This research proposes a methodological approach to understand what narrations related to the application and its users are disseminated on YouTube, an essential platform for spreading narratives about various topics and sharing content. A quantitative and qualitative analysis of the most influential YouTube videos on Tinder was carried out using digital methods. The results show that they portrayed the online dating stages, such as creating a profile, matching, and meeting, reflecting on and upholding the controversies associated with the application. The creation of videos was primarily driven by curiosity about Tinder and the need to provide appealing content to the audience.

\section{Keywords (Source: Unesco Thesaurus)}

Mobile phone; communication; online dating; Tinder; YouTube; videos. 


\section{Introdução}

Os serviços de dating mediados não são algo exclusivo da atualidade. Por um lado, há uma adaptação de serviços existentes e, por outro, a oferta de novos serviços, acompanhando a evolução no âmbito das tecnologias de informação e comunicação. Se anteriormente o uso de tais serviços estava rodeado de algum secretismo, tem-se assistido a uma tentativa de normalização destes, posicionando-se como soluções adicionais às redes sociais e a lugares tradicionais como meio para conhecer novas pessoas (LeFebvre, 2017), potenciadas pelas características da internet e expandindo as redes de contacto. Porém, tendo em conta as circunstâncias que rodeiam a temática do online dating, tem sido desafiante para os seus utilizadores exporem-se e partilharem as suas experiências dissociando-se das representações sustentadas pelos media e do estigma ainda existente. Nesse contexto, as plataformas de social media não só se posicionam como um meio através do qual é possível obter informações sobre determinado tema, numa perspetiva pessoal da experiência, mas também vieram permitir aos utilizadores tal exposição e partilha.

Assim, o objetivo do presente trabalho é identificar narrativas relativas ao universo Tinder, no YouTube, definindo-se como pergunta de partida orientadora: quais as principais narrativas disseminadas em vídeos sobre o Tinder, no YouTube?

\section{Online dating: contextualização}

O conjunto de meios de comunicação digitais mediados aos quais os indivíduos podem recorrer, com o objetivo de conhecer outros e formarem relacionamentos, tem-se diversificado nas últimas décadas, acompanhando a evolução das tecnologias de informação e comunicação. Nesse âmbito, e com o surgimento das tecnologias móveis, nomeadamente os smartphones, o paradigma, no que ao online dating diz respeito, modificou-se. Num cenário em que os sites de encontros predominavam, evoluiu-se para uma realidade em que as aplicações (apps) se apresentam como a ferramenta dominante (Lopez e Vogel, 2019). Adicionalmente, e num mercado em constante crescimento, estamos perante um conjunto de aplicações específicas de online dating, que, ao longo dos anos, se têm diferenciado e diversificado (Albury 
et al., 2017) e cujo número de utilizadores tem aumentado. Estima-se que, no total, e relativamente ao ano de 2019, existissem 219,77 milhões de utilizadores de serviços de online dating no mundo, cujas idades se concentrariam entre 25 e 34 anos (43\%) e entre 35 e 44 anos (25\%) (Statista, 2019).

Em termos gerais, o processo de online dating desenvolve-se em três fases: 1) a criação do perfil, que consiste na construção do perfil, a auto(re) apresentação do utilizador, 2) a correspondência, que se refere ao momento no qual o utilizador define as suas preferências de procura em função de um conjunto de filtros/requisitos disponíveis e 3) a descoberta, que reside no momento em que os utilizadores começam a comunicar entre si (Markowitz, Hancock e Tong, 2018). Dependendo das plataformas, algumas fases podem ser mais extensas do que outras e estar condicionadas a regras mais rígidas. Existem, por exemplo, aquelas que exigem mais informação do que outras na criação do perfil ou que seja a mulher a tomar a iniciativa na fase da descoberta.

Apesar de o número crescente de aplicações e de indivíduos que as utilizam, o ato de recorrer a serviços de online dating e, por conseguinte, os seus utilizadores, ainda são alvo de estigma. Na base do julgamento está, entre outros aspetos, um conjunto de evoluções históricas, económicas e culturais no que ao namoro diz respeito, assim como a introdução de diferentes comportamentos, não só, mas também, proporcionados pela tecnologia, destacando-se práticas como hooking up ou sexo casual (LeFebvre, 2017).

O estigma, referente ao online dating, tem-se apoiado em características pessoais dos utilizadores, nas motivações para recorrer a tais serviços, entre as quais se encontram infidelidade e sexo casual (Timmermans, De Caluwé e Alexopoulos, 2018), num conjunto de comportamentos online, como o ghosting (Turkle, 2015), assim como no formato e na lógica de funcionamento das próprias aplicações (Duguay, 2016; Sepúlveda e Vieira, 2019a). Nesse âmbito, influi na construção de tal estigma, como os utilizadores são apresentados, remetendo para uma ótica de comercialização, assim como a criação de um ambiente estéril relativamente ao assumir compro- 
missos, devido à quantidade de perfis/pessoas disponíveis (Heino, Ellison e Gibbs, 2010). Também o suportam características inerentes à mediação digital, apoiadas em aspetos como a construção de perfis falsos, o engano no decorrer do processo e os desafios de expressão em ambientes mediados ou comportamentos proporcionados por estes. Não obstante, esforços têm sido realizados no sentido da desestigmatização e da normalização, seja por ações das plataformas, ao imporem regras na construção dos perfis, procurando evitar a criação de perfis falsos, ao incentivarem os utilizadores a denunciarem situações ou utilizadores com atitudes menos próprias e consideradas desagradáveis, seja por partilharem testemunhos positivos sobre as suas experiências (veja-se por exemplo o canal do YouTube Badoo e a série Dating stories). Nesse contexto, os utilizadores de apps de online dating são figuras-chave na construção de representações relativas ao online dating, nomeadamente através da partilha de experiências entre as suas redes informais (Finkel et al., 2012).

Entre as apps disponíveis no mercado, o Tinder tem-se destacado pela sua popularidade, características e funcionalidades distintivas, e pelo número acumulado de utilizadores. Tais fatores levaram a que a aplicação tivesse sido alvo de interesse da imprensa generalista, com consequências nas representações sobre ela e os seus utilizadores (Sepúlveda e Vieira, 2019b) assim como a que tivesse registado um corpo crescente de investigação académica, reunindo contributos em áreas como autoapresentação (Ward, 2016; Vieira e Sepúlveda, 2017), motivações (Sumter, Vandenbosch e Ligtenberg, 2016; Timmermans e De Caluwé, 2017; Ranzini e Lutz, 2017) ou funcionamento e autenticidade (David e Cambre, 2016; Duguay, 2016).

\section{Tinder: breve caracterização}

O Tinder apresenta-se como "a aplicação mais popular do mundo para conhecer novas pessoas" (Tinder, s. d.), enquadrando-se no conjunto de aplicações de online dating que têm surgido para smartphones. Foi criada em 2012, estando atualmente disponível em mais de 190 países, contabilizando $57 \mathrm{mi}$ lhões de utilizadores, entre os quais já ocorreram 20 mil milhões de matches (Iqbal, 2019). Opera dentro da lógica fremium, oferecendo uma versão gra- 
tuita, mas limitada em termos de funcionalidades, e versões premium, com diferentes pagamentos mensais, assim como a possibilidade de o utilizador realizar pagamentos esporádicos para aceder a funcionalidades específicas.

A utilização exige a criação de um perfil, cuja operação pode ser realizada através do Facebook, do Google (e-mail) ou de um número de telemóvel. O utilizador, que deverá ter no mínimo 18 anos, construirá a sua autoapresentação com recurso à informação textual (nome, idade, escola, trabalho e "biografia", espaço com 500 caracteres), informação visual e ligação a redes sociais online como o Instagram. Estabelecerá também critérios de procura de outros utilizadores: género, idade e distância da qual se encontram. $\mathrm{O}$ funcionamento por geolocalização é umas das características do Tinder, sendo um exemplo das denominadas location-based real time dating app (David e Cambre, 2016).

Como resultado de tais critérios, a aplicação mostra individualmente perfis de outros utilizadores. A lógica de funcionamento está assente no ato de deslizar (swipe) um a um, tais perfis. Deslizar para a direita (swipe ri$g h t$ ) indica que gosta, deslizar para a esquerda (swipe left) indica que não gosta, sendo forçada a tomada da decisão para poder ver o perfil seguinte. Essa lógica binária (gostar ou não gostar) conferiu awareness à aplicação, posicionando-se como um elemento diferenciador do conjunto de plataformas disponíveis no mercado (Hetsroni, Tuncez e Özdemir, 2019). Ao existir um interesse comum entre dois utilizadores (ambos deslizaram para a direita o perfil do outro), dá-se o match (correspondência) e podem começar a comunicar entre si.

No Tinder, a informação visual é privilegiada em face da textual (Orosz et al., 2016), na medida em que os perfis são apresentados com base nas fotografias, ocupando estas o comprimento do ecrã do telefone e exigindo ao utilizador que procure pela biografia textual, sempre e quando esta exista. Tal característica, aliada às informações através das quais os utilizadores podem criar os seus perfis, como o podem fazer, a lógica de avaliação dos perfis (deslizar), o secretismo relativo ao algoritmo por trás da sugestão 
de perfis e o facto de ser (re)conhecida por encorajar sexo casual (Timmermans e Courtois, 2018) têm levantado várias questões sobre a sua utilização.

\section{YouTube como forma de expressão}

Num ambiente social mediado, outro dado que não se pode ignorar é o contínuo crescimento das redes sociais online, nomeadamente, como espaço de partilha de opiniões e, como alternativa, espaço reservado anteriormente aos media tradicionais.

Nesse contexto, nas últimas décadas, também a partilha de aspetos relativos à vida privada e concretamente relativos à intimidade sofreu transformações. Com a popularização da internet e das redes sociais online, as barreiras entre a vida pública e a privada tornaram-se mais ténues, acompanhando a ideia de uma internet relacional (Lievrouw, 2012). No âmbito dos media sociais online como espaço para a partilha de conteúdo na construção de narrativas sobre determinadas temáticas, impõe-se o estudo de como aquelas relativas ao online dating são comunicadas e por quem. Entre o conjunto de plataformas de media sociais, o YouTube tem-se destacado pelo número crescente de utilizadores, acumulando mais de dois mil milhões de utilizadores ativos mensalmente, estando disponível em 80 idiomas e somando mil milhões de horas de visualizações diárias (YouTube, s. d.). Na plataforma, os utilizadores podem publicar vídeos, visualizar conteúdo publicado por outros e reagir a este através de ações como gostar, não gostar, comentar ou partilhar.

O crescimento e a integração do formato vídeo em outras plataformas remete para a sua importância como recurso e forma de expressão na construção de narrativas quanto a temáticas diversificadas e como formato eleito pelos utilizadores para a sua disseminação (Burgess e MatamorosFernández, 2016; Moe, 2019). Assim, a análise do conteúdo de vídeos, como objeto social visual cada vez mais presente, é fundamental na compreensão da construção de narrativas sobre as mais variadas dinâmicas sociais entre as quais se incluem aquelas referentes ao online dating. Tendo em conta o número de utilizadores de apps de online dating e da importância atribuída ao YouTube, o presente trabalho propôs-se estudar as narrativas 
construídas em vídeos relativos à aplicação Tinder. Para tal, foi definida a seguinte pergunta de investigação: quais as principais narrativas disseminadas em vídeos sobre o Tinder, no YouTube? Estipularam-se como objetivos 1) identificar os vídeos mais influentes e 2) caracterizá-los de forma quantitativa e qualitativa, identificando temáticas abordadas.

\section{Metodologia}

\section{Recolha de dados}

$\mathrm{O}$ artigo resulta de um conjunto de ações, ferramentas e pressupostos que visam desenvolver métodos digitais adequados (Omena, 2019) para o estudo de plataformas de social media. A recolha e a obtenção de dados provenientes de tais plataformas é um desafio crescente para o investigador, seja pela limitação do acesso às Application Programming Interface (API), seja pelo custo inerente às recolhas. Nesta investigação, recorreu-se ao YouTube Data Tools, especificamente ao módulo "Video Network", que recolhe vídeos relacionados através da API v3 do YouTube (Rieder, 2015). Definiu-se como query de pesquisa a palavra "Tinder" e estabeleceu-se o ranking como "relevant". A recolha, realizada em setembro de 2019, não foi limitada temporalmente, já que o objetivo do estudo não recaía sobre um período específico e, por razões semelhantes, não se definiu idioma ou restringiu região de pesquisa.

O ficheiro resultante da recolha, foi importado para o software Gephi com o objetivo de visualizar o grafo relativo à rede de vídeos obtida. No grafo gerado, os vídeos eram representados por círculos (nós) e as interações, dentro da lógica de funcionamento do YouTube (recomendar vídeos ou vídeos serem recomendados), eram representadas por linhas (arestas). Para além da visualização da rede de vídeos obtida, foi possível, através da aplicação de um conjunto de estatísticas e algoritmos, identificar comunidades dentro da rede, identificar vídeos-chave no que à influência na rede gerada diziam respeito e, posteriormente, analisar métricas e temáticas abordadas neles. Nesse contexto, os vídeos foram ordenados pelo seu grau de influência, com foco na análise tanto quantitativa como qualitativa, nos 10 vídeos mais influentes. 
Num primeiro momento, foi realizada uma análise quantitativa das métricas subjacentes à interação dos utilizadores relativamente aos vídeos em termos de engagement (número de visualizações, comentários, gosto, não gosto). Estas métricas facultaram pistas sobre a viralidade dos vídeos, que podem estar assente não só em aspetos como o poder emocional (Dobele et al., 2007) ou em conteúdos provocadores, sexuais e violentos (Porter e Golan, 2002), mas também nos protagonistas e nas audiências (Vollmer e Precourt, 2008). Num segundo momento, foi realizada uma análise temática (Boyatzis, 1998; Braun e Clarke, 2016) recorrendo a uma abordagem indutiva, permitindo que o conteúdo dos vídeos determinasse as temáticas ao explorar as suas narrativas. Tal análise foi realizada contemplando um conjunto de etapas que envolviam: 1) familiarização com os dados, através do visionamento dos vídeos e realização de anotações, 2) codificação dos dados, apontando momentos dos vídeos e criando códigos para categorizar o respetivo conteúdo, 3) criação de temas através da identificação de padrões entre os códigos, 4) revisão dos temas, que implicou voltar a visualizar os vídeos no sentido de averiguar se os temas eram representativos do seu conteúdo, e 5) designação de temáticas comuns, percebendo como estas ajudavam a retratar o conteúdo e assuntos abordados nos vídeos.

Apesar de a unidade de análise serem vídeos, não foi realizada uma análise do ponto de vista estético ou da composição, ao não ser o objetivo da investigação.

No quadro ético, é de referir que o YouTube não obriga a que os canais tenham nomes reais e podem ser definidos níveis de privacidade das publicações. Quanto aos dados dos vídeos, estes foram recolhidos com a ferramenta YouTube Data Tools que opera através da API disponibilizada pelo próprio YouTube. Do conjunto de resultados, constatou-se que a amostra era composta por vídeos pertencentes a canais com grandes audiências, cujos autores apelavam à interação (gostar, comentar, partilhar ou subscrever canal), fatores que remetem para baixas expectativas relativas à privacidade. Nesse contexto, os títulos dos vídeos e os nomes dos canais não foram anonimizados seguindo procedimentos semelhantes aos de outros investigadores (Rieder et al., 2018; Segarra-Savedra e Hidalgo-Marí, 2018; Burgess e Matamoros-Fernández, 2016). 


\section{Resultados}

\section{Caracterização geral da rede}

A rede de vídeos obtida era composta por 496 nós (vídeos) e 3.850 arestas (ligações). Ao grafo gerado, representativo da rede (Figura 1), aplicou-se o algoritmo modularidade. $\mathrm{O}$ objetivo foi quantificar o número de comunidades que formavam a rede, em função dos assuntos comuns tratados nos vídeos que as compunham (Hammer, 2018). Identificaram-se 17 comunidades. Entre estas comunidades eram abordadas temáticas tão diversificadas como a partilha de experiências resultantes do uso da aplicação - comunidade representada no centro da imagem em cor rosa - englobando abordagens como a construção de perfis, o processo de deslizar, a leitura de mensagens ou o relato de paródias. Informações sobre funcionamento - comunidade representada no centro da imagem a cor azul — em que se incluíam dicas sobre como iniciar uma conversa ou como destacar o perfil e conteúdos partilhados pelos media - comunidade representada na rede a amarelo claro.

Estas três comunidades, que representavam respetivamente $23,8 \%$, $17,5 \%$ e 6,9\% do total dos vídeos na rede, continham maioritariamente vídeos em inglês. Não obstante, também se verificaram comunidades onde a maior parte dos vídeos eram em outros idiomas, tais como português, terceira maior comunidade $(7,4 \%)$, francês $(5,6 \%)$, espanhol $(4,8 \%)$, alemão $(5,23 \%)$, assim como contribuições pontuais em macedónio, vietnamita, japonês ou turco. O parâmetro "idioma” era denominador comum nas comunidades, ligando-se a outras por meio de vídeos que funcionavam como pontes entre elas (Burgess e Matamoros-Fernández, 2016). A presença de vídeos de diferentes idiomas remeteu para a abrangência do Tinder em termos geográficos e para a diversidade na origem de conteúdos, funcionando igualmente como um indicador da atenção conferida a tal temática.

\section{Top 10 vídeos mais influentes}

Ao observar a Figura 1, é possível constatar a existência um conjunto de vídeos que se destaca dos restantes, por o círculo que os representa ser de maior dimensão. Tal, é resultado da aplicação do algoritmo betweenness centrality no Gephi. Este algoritmo mede a influência do vídeo na rede, atribuindo- 
lhe um valor em função da centralidade e da frequência com que ele aparece no caminho mais curto entre os restantes vídeos (Heymann, 2015). Na Tabela 1, reúnem-se os dados relativos aos 10 vídeos mais influentes no total da rede em análise, de acordo com os dados disponíveis. Em termos de datas, compunham a amostra vídeos de $2015(\mathrm{n}=1), 2016(\mathrm{n}=1), 2017$ $(\mathrm{n}=2), 2018(\mathrm{n}=4)$ e $2019(\mathrm{n}=1)$. Estes dados são um exemplo da intemporalidade dos conteúdos publicados online, da contínua disponibilidade de acesso, disseminação e de como a aplicação Tinder é, ao longo de um intervalo temporal de quatro anos, alvo da atenção e objeto para a produção de conteúdo.

\section{Figura 1. Comunidades que compõem a rede de vídeos Tinder no YouTube}
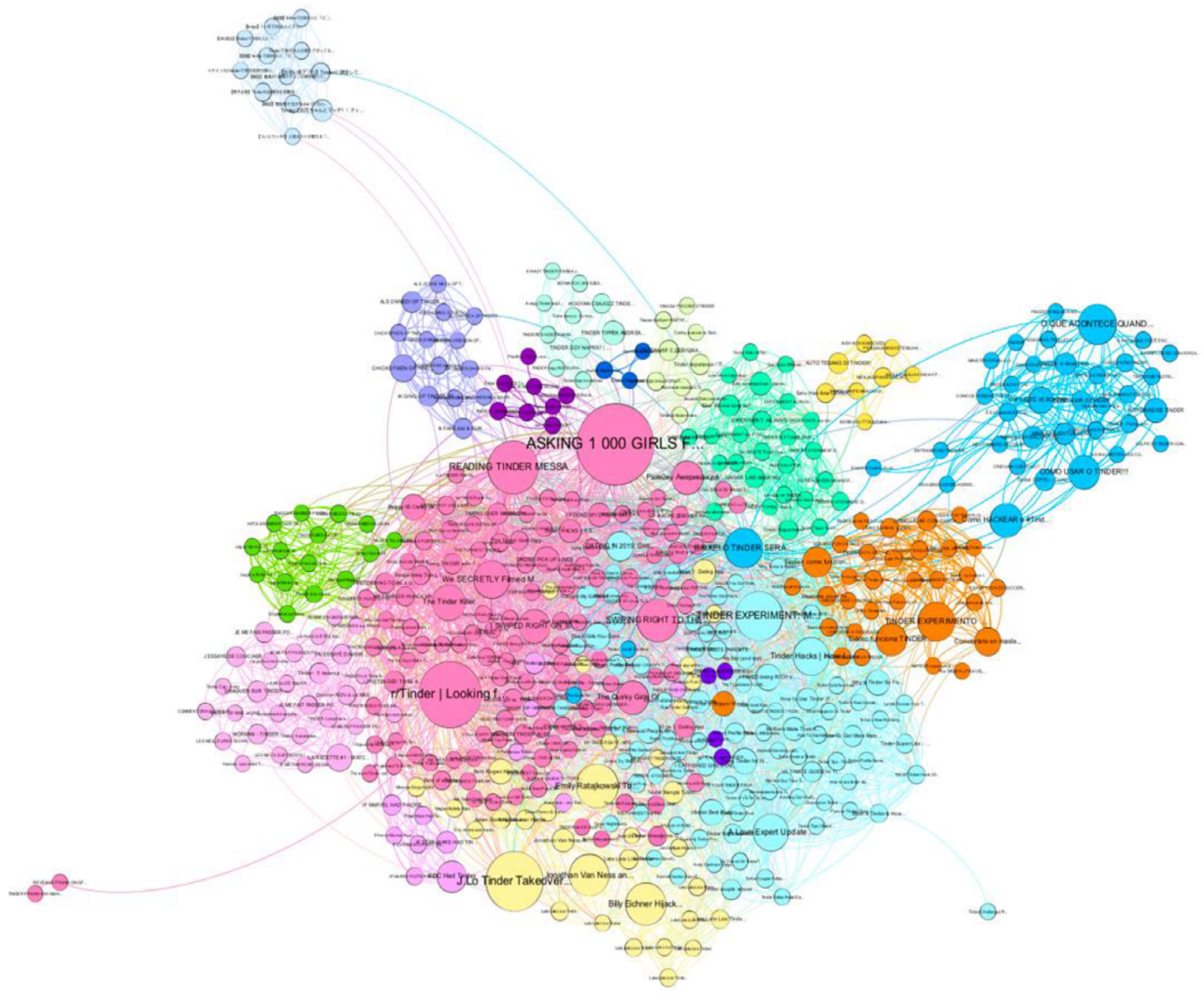

A cor define as diferentes comunidades. 0 tamanho dos nós é estabelecido em função do valor atribuído de betweenness centrality.

Fonte: elaboração própria dos autores. 
Com respeito ao vídeo mais visualizado, e de acordo com os dados obtidos no momento da recolha, este foi "J.Lo Tinder Takeover | Swipe Sessions | Tinder", que contabilizava 7.534.154 visualizações. Participava nele a cantora Jennifer Lopez, cujo canal do YouTube somava, no momento da análise, mais de 12 milhões de subscritores.

\section{Tabela 1. Top 10 vídeos mais influentes ordenados por ordem decrescente em função do valor atribuído de betweenness centrality}

\begin{tabular}{|c|c|c|c|c|c|c|c|}
\hline Título & 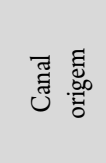 & 㞼 & 胥 & 율 & 总总 & 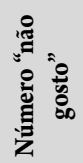 & 율 \\
\hline $\begin{array}{l}\text { Asking } 1000 \text { girls for sex } \\
\text { (Tinder experiment) }\end{array}$ & whatever & $13 / 12 / 2016$ & $11: 54$ & 6.278 .876 & 55.117 & 8.903 & 5.946 \\
\hline $\begin{array}{l}\text { r/tinder | looking for a right } \\
\text { hand man }\end{array}$ & EmKay & $18 / 06 / 2019$ & $15: 25$ & 379.341 & 15.276 & 227 & 952 \\
\hline $\begin{array}{l}\text { J.Lo Tinder takeover } \mid \text { swipe } \\
\text { sessions } \mid \text { Tinder }\end{array}$ & Tinder & $11 / 07 / 2018$ & $02: 20$ & 7.534 .154 & 4.270 & 2.693 & 97 \\
\hline $\begin{array}{l}\text { Reading Tinder messages! I } \\
\text { made a Tinder for } 20 \text { minutes } \\
\text { and this is what happened... }\end{array}$ & $\begin{array}{c}\text { Alana } \\
\text { Arbucci }\end{array}$ & $13 / 06 / 2018$ & $14: 43$ & 1.764 .643 & - & - & 1.667 \\
\hline $\begin{array}{l}\text { Tinder experiment: men vs } \\
\text { women }\end{array}$ & whatever & $07 / 12 / 2015$ & $01: 59$ & 3.591 .735 & 33.004 & 2.288 & 6.311 \\
\hline $\begin{array}{l}\text { Emily Ratajkowski takes } \\
\text { over for a Tinder user | swipe } \\
\text { session } \mid \text { Tinder }^{2}\end{array}$ & Tinder & - & - & 3.813 .798 & 6.027 & 1.332 & 299 \\
\hline $\begin{array}{l}\text { Swiping right to the first } 100 \\
\text { people on Tinder (this is what } \\
\text { happened) }\end{array}$ & $\begin{array}{l}\text { Kirsty } \\
\text { Belle }\end{array}$ & $20 / 12 / 2018$ & 09:11 & 64.370 & 1.705 & 215 & 103 \\
\hline $\begin{array}{l}\text { Billy Eichner hijacks a stranger } \\
\text { s Tinder | Vanity Fair }\end{array}$ & $\begin{array}{l}\text { Vanity } \\
\text { Fair }\end{array}$ & $09 / 03 / 2017$ & $08: 29$ & 2.052 .192 & 26.522 & 1.828 & 1.878 \\
\hline $\begin{array}{l}\text { Jonathan Van Ness and Antoni } \\
\text { Porowski swipe for a Tinder } \\
\text { user } \mid \text { swipe session | Tinder }\end{array}$ & Tinder & $03 / 10 / 2018$ & $03: 42$ & 1.834 .268 & 9.005 & 251 & 190 \\
\hline $\begin{array}{l}\text { O que acontece quando uma } \\
\text { menor de idade entra no } \\
\text { Tinder? }\end{array}$ & $\begin{array}{l}\text { Segunda } \\
\text { Animada }\end{array}$ & $14 / 12 / 2017$ & $08: 34$ & 1.905 .055 & 165.472 & 2414 & 6.786 \\
\hline
\end{tabular}

Fonte: elaboração própria dos autores.

2 Não foi possível realizar análise temática do vídeo, da data de publicação e da duração uma vez que, após a recolha dos dados, o conteúdo deixou de estar visível. 
O segundo vídeo mais visualizado, "Asking 1000 girls for sex (Tinder experiment)", correspondia ao que detinha o valor de centralidade na rede mais elevado, e somava 6.278 .876 visualizações. $O$ terceiro vídeo mais visualizado, "Emily Ratajkowski Takes Over for a Tinder User|Swipe Session | Tinder”, acumulava 3.813.798 visualizações e, à semelhança do mais visto, também contava com a participação de uma figura pública, a modelo Emily Ratajkowski, cujo perfil do Instagram somava mais de 25 milhões de seguidores. A estratégia de incluir no vídeo indivíduos que gozam de notoriedade ao serem figuras públicas ou que geram interesse em algo - influencers - e que acumulam elevadas audiências nos media sociais online, tem impacto no alcance e consequente visualização dos vídeos na medida em que tais figuras têm a capacidade de mobilizar um grande número de pessoas (Vollmer e Precourt, 2008).

O vídeo menos visualizado, "Swiping right to the first 100 people on Tinder (this is what happened)” contabilizava 64.370 visualizações. Porém, posicionava-se como mais influente comparativamente a vídeos da amostra com um maior número de visualizações ou que eram protagonizados por figuras públicas e de qual é exemplo o vídeo "Billy Eichner hijacks a strangers Tinder | Vanity Fair”.

O tempo acumulado de duração dos vídeos em análise era de $1 \mathrm{~h} 16 \mathrm{~m} 17 \mathrm{~s}$ e a média de duração dos mesmos $08 \mathrm{~m} 29 \mathrm{~s}$. O vídeo mais longo, "r/Tinder | Looking for a Right Hand Man", com 15m25s, correspondia ao segundo mais influente. Já o vídeo mais curto, que durava $2 \mathrm{~m} 20 \mathrm{~s}$, era o mais visualizado "J.Lo Tinder Takeover | Swipe Sessions | Tinder". Embora o tempo de duração de um vídeo condicione a captação de interesse e visualização, outros fatores como a sua natureza, temática, abordagem, conteúdo, protagonistas e o idioma podem ter resultados no seu alcance e no seu grau de influência.

Os dados relativos ao engagement dos utilizadores, referentes aos vídeos da amostra em análise, indicaram que o menos influente, "O que acontece quando uma menor de idade entra no Tinder?", foi aquele que acumulou o maior número de reações alusivas à ação "gostar" (165.472). 
Por cada visualização, 8,7 \% dos utilizadores gostou do vídeo. O segundo vídeo que acumulava o maior número de gostos (55.117), "Asking 1000 girls for sex (Tinder experiment)", correspondia ao mais influente da amostra e, por cada visualização, correspondeu 0,8 \% "gosto". O terceiro vídeo que acumulou um maior número de "gosto", “Tinder experiment: men vs women", posicionava-se ao meio da tabela, contabilizando 33.004 "gosto" e, de acordo com o número de visualizações (3.591.735), obteve reações positivas por parte de $0,9 \%$ dos utilizadores que o viram.

Quanto aos vídeos que acumularam o maior número de "não gosto", estes foram "Asking 1000 girls for sex (Tinder experiment)", com 8.903 "não gosto", seguindo-o "J.Lo Tinder Takeover | Swipe Sessions | Tinder", com 2.693, e o vídeo "O que acontece quando uma menor de idade entra no Tinder?”, com 2.414 reações negativas.

Em função das definições de cada canal, os utilizadores podem ou não comentar os vídeos e, entre aqueles pertencentes à amostra, todos acumulavam comentários. Entre esses, aquele que mais comentários acumulava foi o vídeo "O que acontece quando uma menor de idade entra no Tinder?", somando 6.786. Por cada utilizador que visualizou o vídeo $0,1 \%$ comentou-o. Já o vídeo menos comentado foi "J.Lo Tinder Takeover | Swipe Sessions | Tinder”, com 97 reações.

$\mathrm{Na}$ origem dos vídeos, estão canais de categorias tão diferentes como media (Vanity fair), comédia (whatever, Em Kay, Segunda Animada), beleza (Kirsty Belle) ou entretenimento, como o canal da aplicação em estudo (Tinder). O propósito dos vídeos também era de diferente natureza, por exemplo, experiências sociais, maquilhagem, leitura de conteúdos de outras plataformas ou que agrupavam temáticas tão variadas como a arte ou a política. Apesar da heterogeneidade e da multiplicidade de canais, o Tinder era o ponto em comum, apontando tal factor, e em termos gerais, para o grau de imiscuidade da plataforma, em diferentes perfis e audiências.

\section{Construção do perfil e autenticidade}

Um dos temas abordados nos vídeos foi o processo de construção do perfil. No decorrer destes, os utilizadores questionavam-se sobre que fotografias 
escolher, chegando inclusive a serem verbalizados pensamentos referentes ao que elas podiam transmitir sobre eles, as perceções que os outros utilizadores podiam formular e, consequentemente, como podiam ser julgados, baseado em pormenores como a roupa, o que estavam a fazer ou o contexto da foto. Tais aspetos também eram refletidos através da opinião de terceiros, apresentados como especialistas ou figuras públicas, que integravam determinados vídeos, nos quais escrutinavam o perfil do protagonista anónimo. São exemplo os vídeos "Jonathan Van Ness and Antoni Porowski swipe for a Tinder user | swipe session | Tinder" e "J.Lo Tinder Takeover | Swipe Sessions | Tinder”, publicados pelo canal do Tinder. Neste último, a cantora faz também considerações negativas sobre os perfis sugeridos em função de parâmetros como a idade. Apesar de ser o vídeo mais visualizado, foi o segundo menos gostado.

No contexto da construção da autoapresentação, a escolha das fotografias refletia critérios estéticos, nomeadamente se o utilizador gostava de se ver, revelando-se a autoestima um importante indicador (Ranzini e Lutz, 2016), sendo as fotografias escolhidas com base na impressão que pretendiam causar nos outros, de qual é exemplo o vídeo "Reading Tinder messages! I made a Tinder for 20 minutes and this is what happened..., e considerando também a probabilidade de match.

Quanto ao campo biográfico de 500 caracteres, esse recurso é pouco referido e, quando o é, é no sentido de os protagonistas não desejarem incluir informação, reforçando a importância atribuída à componente visual na construção dos perfis, em detrimento da textual (Vieira e Sepúlveda, 2017).

No processo de criação do perfil, foram também mencionadas questões relacionadas com a autenticidade, fosse pela opção não só de escolher fotografias editadas, mas também em momentos nos quais os protagonistas se questionavam se deviam indicar o nome verdadeiro, em que forneciam informação falsa como a idade, indicando serem mais velhos para poderem estar na app, ou inclusive vídeos em que foram criados perfis falsos como o vídeo “Tinder experiment: men vs women”. Dependendo do motivo (fazer uma experiência, curiosidade, alertar) e da decisão inerente, ficava clara a 
ideia de como era relativamente simples construir um perfil com informação errónea, fortalecendo construções subjacentes ao engano em ambientes e práticas relacionadas com o online dating.

Adicionalmente, e embora em alguns vídeos fosse possível visionar o processo de download da aplicação, em nenhum momento dos vídeos os utilizadores demonstraram procurar saber como a aplicação funcionava, mesmo quando afirmavam não a conhecerem ou que a estavam a utilizar pela primeira vez. Tal aspeto refletia-se numa utilização praticamente intuitiva, mas que os levava a colocar questões sobre o funcionamento, sobre que informação sua era apresentada aos outros utilizadores, e a críticas, argumentando que a aplicação era complicada ou que lhes obrigava a gastar dinheiro para poderem continuar a usar.

\section{Deslizar, corresponder e comunicar}

O processo de deslizar perfis (swipe) foi retratado com frequência nos vídeos. Através desse processo, foi novamente dado enfoque à componente visual. As decisões dos utilizadores eram tomadas em função da visualização das fotografias e muitas vezes em função da primeira. Se existissem dúvidas e se quisessem saber mais antes de tomar a decisão (aceitar ou rejeitar perfil), visualizavam as restantes fotografias ou liam a biografia, quando esta existia. Em tal processo, muitas vezes em função do contexto do vídeo, a maior parte dos perfis sugeridos era deslizada para a direita, sem consultar qualquer informação e só depois, após o match, é que o utilizador os vi-a em detalhe. Tal comportamento, descrito por LeFebvre (2017) como shotgun approach, é em parte realizado no sentido de o utilizador alcançar o maior número de matches. No vídeo "Swiping right to the first 100 people on Tinder (this is what happened)", o número de matches alcançados é sinónimo de popularidade e, em outros, o objetivo principal é atingir um determinado número.

Quando o número de perfis deslizados atingia o limite, determinado em função do algoritmo da app, era sugerido ao utilizador que pagasse para prosseguir. Nesse mesmo contexto, os utilizadores também se questionavam como é que podiam saber quem tinha gostado deles - funções reservadas 
às versões que exigem pagamento, remetendo para questões relacionadas com o desconhecimento do funcionamento (Sepúlveda e Vieira, 2019a).

Para além de restrições relacionadas com o funcionamento, como o limite relativo ao número de swipes possíveis, também foram abordadas questões relacionadas com o funcionamento do algoritmo em função do género. Tal facto é visível no vídeo “Tinder experiment: men vs women”, em que foram criados dois perfis (homem e mulher) falsos, supostamente semelhantes entre si em termos de informação visual e textual, mas com resultados diferentes da interação no que ao número de matches e mensagens recebidas dizia respeito. Nessas métricas, o perfil correspondente ao género feminino tinha obtido números mais elevados, sendo argumentada a ideia de que a app era díspar em função do género. Ainda no âmbito do género, em nenhum vídeo, são considerados outros para além do binarismo masculino-feminino, sendo a utilização retratada limitada na perspetiva heterogénea, assim como na orientação sexual. Aspetos apontados como limitadores na construção do perfil e na utilização da plataforma (Duguay, 2016).

No processo de descoberta e no contexto da comunicação, as mensagens enviadas/recebidas no Tinder são produto de um ambiente digital mediado. Como resultado, os protagonistas dos vídeos, ao lerem essas mensagens, conferiam entoação e interpretavam-nas. Também se registou, ainda no âmbito das mensagens, mas na componente escrita, a presença de emoji ou gifs como forma de reforçarem determinada ideia.

De uma forma geral, os utilizadores pareciam valorizar as mensagens que continham um comentário ou uma pergunta específica sobre o seu perfil, ou uma piada considerada por eles de bom gosto, em detrimento de mensagens mais generalistas ou que expressassem apenas “olá". É assim reforçada a ideia de que o utilizador se tem de destacar também na forma como inicia a conversa, muitas vezes como resultado da própria lógica de funcionamento da aplicação e da ideia da quantidade de perfis disponíveis e consequente escolha (Heino et al., 2010).

No vídeo mais influente, e que acumulava o maior número de "não gosto”, o propósito era conseguir 1000 matches para enviar a mesma mensa- 
gem a cada um dos utilizadores "Hey, do you want to have sex?", focandose a dinâmica do vídeo na leitura das respostas. $\mathrm{O}$ protagonista principal, um homem, com uma t-shirt com a palavra "slut", contribuía para a construção da ideia de que o Tinder tem sido considerada uma aplicação para sexo (LeFebvre, 2017) assim como formatava a audiência relativamente ao tipo de pessoas que a utilizam.

$\mathrm{Na}$ dinâmica de respostas, e em função destas (positivas ou negativas), o protagonista por vezes parodiava aqueles que tinham respondido, discriminava, chegando a ser ofensivo e refletindo um conjunto de estereótipos, por vezes aliados ao género.

A leitura de mensagens era também o mote do segundo vídeo mais influente. Ao longo dos seus mais de 15 minutos de duração uma voz offlia publicações partilhadas na plataforma Reddit. Entoava a leitura, fazia piadas, assim como, considerações depreciativas sobre as mensagens e/ou sobre os perfis. Tais resultados remetem para uma toxicidade masculina e para o facto de os utilizadores do Tinder estarem sujeitos à humilhação pública em outras redes sociais online (Hess e Flores, 2016).

No processo de troca de mensagens, ficou também patente a ideia de que os utilizadores, após as receberem e as lerem, terem liberdade para não responderem às mesmas, comportamento possível e proporcionado pelos ambientes mediados digitais (Turkle, 2015).

\section{Conclusões}

O Tinder revelou-se ser um objeto de atenção por parte de um conjunto heterogéneo de canais do YouTube e uma temática atrativa para a produção de conteúdo, do qual é reflexo o engagement que os vídeos em análise registaram.

Estes relatavam, na sua maioria, experiências ao utilizar a app nomeadamente na criação do perfil, no processo de deslizar e na comunicação entre utilizadores. No decorrer da utilização, também foram abordadas diferentes funcionalidades e apontadas limitações. Foi possível ver retratadas polémi- 
cas associadas à app e que têm estado na origem do ainda existente estigma social, muitas vezes articuladas à própria lógica de funcionamento e regras. Nesse contexto, os utilizadores contribuíam para a construção de representações aliadas à utilização da app, através de um conjunto de comportamentos e julgamentos sobre os outros e sobre si. Não obstante, é necessário ter em conta o contexto, a natureza e os objetivos dos vídeos publicados.

Entre os vídeos mais influentes, não se registou a partilha de testemunhos de pessoas que se tenham conhecido no Tinder, ex-utilizadores ou atuais, com um foco positivo e nas potencialidades base das aplicações de online dating. $\mathrm{O}$ Tinder foi retratado como uma app em que é fácil criar um perfil, inclusive como é possível criar um perfil falso, à qual está associada um alto grau de entretenimento e curiosidade, duas das motivações apontadas pelos utilizadores para usar (Timmermans e De Caluwé, 2017).

A análise quantitativa permitiu concluir que a influência em termos de centralidade na rede é independente do engagement dos utilizadores assim como, do número de visualizações acumulado. $\mathrm{O}$ vídeo mais influente não era o mais visto, gostado ou comentado. Tais dados remetem para a lógica de recomendação da plataforma YouTube e o seu funcionamento. Nesse sentido, seria interessante, em estudos futuros, compreender em mais detalhe o papel do YouTube como plataforma que aloja e recomenda vídeos. Adicionalmente e numa outra abordagem, uma análise dos comentários aos vídeos ajudaria no sentido de compreender como eles eram recebidos e o seu impacto.

\section{Referências}

Albury, K., Burgess, J., Light, B., Race, K. e Wilken, R. (2017). Data cultures of mobile dating and hook-up apps: Emerging issues for critical social science research. Big Data \& Society, 4(2), 1-11. DOI: https://doi.org/10.1177/2053951717720950

Boyatzis, R. E. (1998). Transforming qualitative information: Thematic analysis and code development. Thousand Oaks: Sage. 
Braun, V. e Clarke, V. (2006). Using thematic analysis in psychology. Qualitative Research in Psychology, 3(2), 77-101. DOI: https://doi.or$\mathrm{g} / 10.1191 / 1478088706 \mathrm{qp} 063$ oa

Burgess, J. e Matamoros-Fernández,A. (2016). Mapping sociocultural controversies across digital media platforms: One week of \#gamergate on Twitter, YouTube, and Tumblr. Communication Research and Practice, 2(1),79-96.DOI: https://doi.org/10.1177/2056305116641976

David, G. e Cambre, C. (2016). Screened intimacies: Tinder and the Swipe Logic. Social Media \& Society, 2(2), 1-11. DOI: https://doi. org/10.1177/ 2056305116641976

Dobele, A., Lindgreen, A., Beverland, M., Vanhamme, J. e Wijk, R. (2007). Why pass on viral messages? Because they connect emotionally. Business Horizons, 50, 291-304. DOI: https://doi.org/10.1016/j. bushor.2007.01.004

Duguay, S. (2016). Dressing up Tinderella: Interrogating authenticity claims on the mobile dating app Tinder. Information, Communication and Society, 20(3), 1- 17. DOI: https://doi.or$\mathrm{g} / 10.1080 / 1369118 X .2016 .1168471$

Finkel, E. J., Eastwick, P. W., Karney, B. R., Reis, H. T. e Sprecher, S. (2012). Online dating: A critical analysis from the perspective of psychological science. Psychological Science in the Public Interest, 13, 3-66. DOI: https://doi.org/10.1177/1529100612436522

Hammer, L. (2018, agosto, 23). Modularity. Recuperado de: https://github.com/gephi/gephi/wiki/Modularity

Heino, R., Ellison, N. e Gibbs, Jennifer. (2010). Relationshopping: Investigating the market metaphor in online dating. Journal of Social and Personal Relationships, 27, 427-447. DOI: https://doi. org/10.1177/0265407510361614 
Hess, A. e Flores, C. (2016). Simply more than swiping left: A critical analysis of toxic masculine performances on Tinder Nightmares. New Media \& Society, 20(3), 1085-1102. DOI: https://doi. org/10.1177/1461444816681540

Hetsroni, A., Tuncez, M. e Özdemir, M. (2019). Stereotypical gender attributions across sexual orientations on Tinder: Evidence from Turkey. Em: A. Hetsroni e M. Tuncez (eds.), It happened on tinder. Reflections and studies on internet-infused dating (pp. 47-62). Amsterdam: Institute of Network Cultures. DOI: https://doi. org $/ 10.36941 / \mathrm{mjss}-2020-0002$

Heymann, S. (2015, 1 março). Betweenness Centrality. Recuperado de: https://github.com/gephi/gephi/wiki/Betweenness-Centrality

Iqbal, M. (2019, 27 fevereiro). Tinder Revenue and Usage Statistics (2018). Recuperado de: https://www.businessofapps.com/data/tinder-statistics/

LeFebvre, L. (2017). Swiping me off my feet: Explicating relationship initiation on Tinder. Journal of Social and Personal Relationships, 35(9), 1205-1229. DOI: https://doi.org/10.1177/0265407517706419

Lievrouw, L. (2012). The next decade in internet time. Information, Communication \& Society, 15(5), 616-638. DOI: https://doi.or$\mathrm{g} / 10.1080 / 1369118 X .2012 .675691$

Lopes, M. e Vogel, C. (2019). Gender differences in online dating experiences. Em: A. Hetsroni e M. Tuncez (eds.), It happened on tinder. Reflections and studies on internet-infused dating (pp. 31-47). Amsterdam: Institute of Network Cultures.

Markowitz, D., Hancock, J. e Tong, S. (2018). Interpersonal dynamics in online dating. Em: Z. Papacharissi (ed.), A Networked self 
and love (pp. 50-61). Nova York: Routledge. DOI: https://doi. org/10.4324/9781315193472-4

Moe, H. (2019). Comparing platform "ranking cultures" across languages: The case of Islam on YouTube in Scandinavia. Social Media + Society, 1-10. DOI: https://doi.org/10.1177/2056305118817038

Omena, J.J. (2019). Métodos Digitais: teoria-prática-crítica. Lisboa: Instituto de Comunicação da Nova.

Orosz, G., Tóth-Király, I., Bőthe, B. e Melher, D. (2016). Too many swipes for today: The development of the Problematic Tinder Use Scale (PTUS). Journal of Behavioral Addictions, 5(3), 518-523. DOI: https://doi.org/10.1556/2006.5.2016.016

Porter, L. e Golan, G.J. (2006). From subservient chickens to brawny men: A comparison of viral advertising to television advertising. Journal of Interactive Advertising, 6(2), 26-33. DOI: https://doi.org/10.1 $080 / 15252019.2006 .10722116$

Ranzini, G. e Lutz, C. (2017). Love at first swipe? Explaining Tinder self-presentation and motives. Mobile Media and Communication, 5(1),80-101.DOI: https://doi.org/10.1177/2050157916664559

Rieder, B. (2015). YouTube data tools. Computer software. Recuperado de: https://tools.digitalmethods.net/netvizz/youtube/

Rieder, B., Matamoros-Fernández, A. e Coromina, O. (2018). From ranking algorithms to 'ranking cultures': Investigating the modulation of visibility in YouTube search results. Convergence: The International Journal of Research into New Media Technologies, 24(1), 50-68. DOI: https://doi.org/10.1177/1354856517736982

Segarra-Saavedra, J. e Hidalgo-Marí, T. (2018). Viralidade e interacción. Análisis del engagement de los diez anúncios más vistos en YouTu- 
be en España en 2016. Ícono 14, 16(1), 47-71. DOI: https://doi. org/10.7195/ri14.v16i1.1069

Sepúlveda, R. e Vieira, J. (2019a). Lógicas de funcionamento do Tinder. Uma análise da aplicação e das perceções dos utilizadores. Teknokultura, 16(1), 75-91.DOI: https://doi.org/10.5209/TEKN.62054

Sepúlveda, R. e Vieira, J. (2019b). Representações da aplicação online dating Tinder na imprensa escrita portuguesa. Estudos em Comunicação, 29, 1-20.

Statista. (2019). Online Dating — worldwide: Statista Market Forecast. Recuperado de: http://www.statista.com/outlook/372/100/online-dating/worldwide

Sumter, S., Vandenbosch, L. e Ligtenberg, L. (2016). Love me Tinder: Untangling emerging adults' motivations for using the dating application Tinder. Telematics and Informatics, 34(1), 67-78. DOI: https:// doi.org/10.1016/j.tele.2016.04.009

Timmermans, E. e De Caluwé, E. (2017). Development and validation of the Tinder motives Scale (TMS), Computers in Human Behavior, 20, 341-350. DOI: https://doi.org/10.1016/j.chb.2017.01.028

Timmermans, E., De Caluwé, E. e Alexopoulos, C. (2018). Why are you cheating on Tinder? Exploring users' motives and (dark) personality traits. Computers in Human Behavior, 89, 129-139. DOI: https://doi.org/10.1016/j.chb.2018.07.040

Tinder. (s. d). O que é o Tinder. Recuperado de: http://www.help.tinder. $\mathrm{com} / \mathrm{hc} / \mathrm{pt} /$ articles/115004647686-O-que-\%C3\%A9-o-Tinder-

Turkle, S. (2015). Reclaiming Conversation: The power of talk in a digital age. Nova York: Penguin. 
Vieira, J. e Sepúlveda, R. (2017). A autoapresentação dos portugueses na plataforma de online dating Tinder. Observatorio. $\left(O B S^{*}\right), 11(3)$, 153-185. DOI: https://doi.org/10.15847/obsOBS11320171150

Vollmer, C. e Precourt, G. (2008). Always on: Advertising, Marketing, and media in an era of consumer control. Nova York, NY: McGraw-Hill Professional.

Ward, J. (2016). What are you doing on Tinder? Impression management on a matchmaking mobile app. Information, Communication and Society, 20(11), 1644-1659. DOI: https://doi. org/10.1080/1369118X.2016.1252412

YouTube. (s. d). Estatísticas do YouTube. Recuperado de: www.youtube. com/about/press/ 